\title{
Antibacterial Screening of some Heterocyclic Compounds Containing Quinoline Nucleus
}

\author{
Shipra Baluja ${ }^{1, a^{*}}$, Nilesh Godvani ${ }^{1, b}$ and Sumitra Chanda ${ }^{2, c}$ \\ ${ }^{1}$ Department of Chemistry, Saurashtra University, Rajkot-360005 (Gujarat), India \\ ${ }^{2}$ Department of Bio Science, Saurashtra University, Rajkot-360005 (Gujarat), India

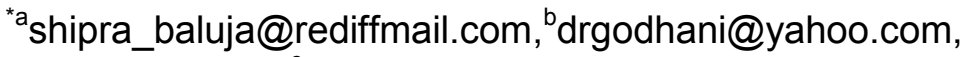 \\ 'svchanda@gmail.com
}

Keywords: Cyanopyridines, Isoxazoles, Vilsmeier-Haack reagent, spectral analysis, DMSO, DMF, antibacterial activity.

\begin{abstract}
In this work, some novel derivatives of Cyanopyridines and Isoxazoles were synthesized using Vilsmeier-Haack reagent and their structures were confirmed by FTIR, ${ }^{1} \mathrm{H}$ NMR and mass spectroscopic methods. The antibacterial activities of these synthesized compounds were studied in DMSO and DMF by agar well diffusion method against some Gram positive and Gram negative bacteria. It is observed that activity depends upon three S: solvent, strain and structure.
\end{abstract}

\section{Introduction}

Quinolines are heterocyclic nitrogen containing basic compounds in which the pyridine ring is electron deficient. Therefore, nucleophilic attack takes place at the 2- and 4-positions. Quinolines and their derivatives are very important compounds because of their wide occurrence in natural products [1,2] and biologically active compounds [3-8]. These derivatives are also used for the preparation of nano- and meso structures having enhanced electronic and photonic properties $[9,10]$ and are known to have applications as pharmaceuticals, agrochemicals and synthetic building blocks [11]. Quinolines have been found to possess antimalarial, anti-bacterial, antifungal, antihelmintic, cardiotonic, anticonvulsant, anti-inflammatory, and analgesic activity [12-18].

Recently, more and more new simple and elegant syntheses of substituted quinolines have been described [19-25]. The synthesis of quinoline derivatives thus continues to be an attractive area of research and the synthesis of various substituted quinolines have been largely described in the literature through different strategies [26-28]. Some of these substituted quinolines are important constituents of several pharmacologically active compounds. A number of biological activities [29-34] have also been associated with quinoline containing compounds such as antiinflammatory, antiallergic, antimalarial, antibacterial, antiproliferative, anticancer and antiparasitic activities.

Thus, aim of the present work is to synthesize some new nitrogen containing heterocyclic compounds and to study their antibacterial activity. Some novel derivatives of cyanopyridine and isoxazole bearing quinoline nucleus are synthesized and their structures were elucidated on the basis of IR, MASS and 1H NMR spectral data. All the compounds have been screened for their invitro biological activity by agar well diffusion method.

\section{Materials and Methods}

The chemicals used in the present work were 4-fluoroaniline, acetic anhydride, sodium bicarbonate, acetophenone, p-methoxy-acetophenone, p-nitro acetophenone, p-aminoacetophenone, $\mathrm{p}$-bromo-acetophenone, m-nitro-acetophenone, $\mathrm{p}$-hydroxy-acetophenone, $\mathrm{p}$-chloroacetophenone, o-hydroxy-acetophenone, malononitrile, ethanol, $\mathrm{POCl}_{3}$, sodium hydroxide, ammonium acetate, sodium acetate, acetic acid, DMF, DMSO, hydroxylamine hydrochloride etc. All these chemicals were purchased from Spectrochem (Mumbai, India) and were of LR grade. These chemicals were used as such for synthesis. 
Test Microorganisms: The microorganisms used in the present study were four Gram positive and four Gram negative bacteria.

Gram positive bacteria: Bacillus cereus ATCC11778, Micrococcus flavus ATCC10240, Staphylococcus epidermidis ATCC12228, and Staphylococcus aureus ATCC29737.

Gram negative bacteria: Proteus mirabilis NCIM2241, Salmonella typhimurium ATCC23564, Citrobacter freundii ATCC10787 and Klebsiella pneumoniae NCIM2719.

Microorganisms were obtained from National Chemical Laboratory (NCL), Pune, India and were maintained at $4^{\circ} \mathrm{C}$ on nutrient agar slants.

\section{Synthesis:}

\section{Cyanopyridines:}

Synthesis of 2-amino-4-(2-chloro-6-fluoroquinolin-3-yl)-6-(4-methoxy phenyl) nicotine nitrile.

Synthesis of $\mathbf{N}$-(4-fluorophenyl)acetamide: Equimolar mixture of 4-fluoroaniline and acetic anhydride $(0.01 \mathrm{M})$ in ethanol was refluxed in oil bath for 2-3 hrs using $\mathrm{H}_{2} \mathrm{SO}_{4}$ as catalyst. The crude product was isolated and crystallized from absolute ethanol.

Synthesis of 2-chloro-6-fluoroquinoline-3-carbaldehyde: $N$ - (4-fluorophenyl) acetamide prepared above was added in a mixture of Vilsmeier-Haack reagent (prepared by drop wise addition of $6.5 \mathrm{ml} \mathrm{POCl}{ }_{3}$ in ice cooled $2 \mathrm{ml} \mathrm{DMF}$ ) and was refluxed for $27 \mathrm{hrs}$ on oil bath. After the completion of reaction, reaction mixture was poured into ice followed by neutralization using saturated aqueous sodium bicarbonate solution. The crude product was isolated by filtration and was crystallized from ethanol.

Synthesis of (2E)-3-(2-chloro-6-fluoroquinolin-3-yl)-1-(4-methoxy phenyl) prop-2-en-1- one: An equimolar solution of 2-chloro-6-fluoroquinoline-3-carbaldehyde and p-methoxy-acetophenone was prepared in ethanol. The solution was made alkaline by $40 \% \mathrm{NaOH}$ aqueous solution. The reaction mixture was stirred for $48 \mathrm{hrs}$. The contents were poured into ice and acidified with $50 \%$ $\mathrm{HCl}$ solution. The obtained crude product was filtered and was crystallized from ethanol.

Synthesis of 2-amino-4-(2-chloro-6-fluoroquinolin-3-yl)-6-(4-methoxyphenyl) nicotine nitrile (NCP-1): To an equimolar mixture of (2E) - 3- (2- chloro- 6-fluoro quinolin-3-yl) -1-(4- methoxy phenyl) prop-2-en-1-one and malononitrile in ethanol, ammonium acetate was added and solution was refluxed for 10-12 hrs in oil bath. The content was poured on crushed ice. The obtained product was filtered, washed with water and crystallized from ethanol. The reaction scheme is given in Fig. 1.

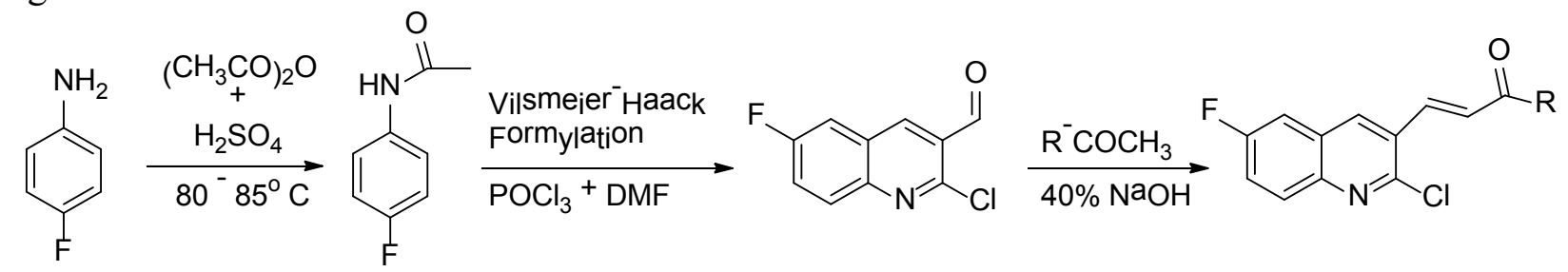

Steps:

[A]

[B]

[C]<smiles>[R]C(=O)/C=C/c1cc2cc(F)ccc2nc1Cl</smiles>

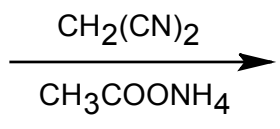<smiles>[R]c1cc(-c2cc3cc(F)ccc3nc2Cl)c(C#N)c(N)n1</smiles>

Figure 1. Reaction scheme for the synthesis of cyanopyridines.

Similarly, ten other substituted cyanopyridines have been prepared. 


\section{Isoxazoles:}

Synthesis of (2E)-3-(2-chloro-6-fluoroquinolin-3-yl)-1-(4-methoxyphenyl) prop-2-en-1- one: It is given above.

Synthesis of 2-chloro-6-fluoro-3-[3-(4-methoxyphenyl) isoxazol-5-yl]quinoline (NISO-1): To an equimolar solution of anhydrous sodium acetate and hydroxylamine hydrochloride in ethanol, minimum amount of hot acetic acid and solution of (2E)-3-(2-chloro-6-fluoro quinolin -3-yl)-1-(4methoxy phenyl) prop-2-en-1-one in ethanol was added. The mixture was refluxed on a water bath for 10 hrs. The product was isolated and recrystallized from ethanol. The reaction is in Fig. 2.<smiles>[R]C(=O)/C=C/c1cc2cc(F)ccc2nc1Cl</smiles>

Figure 2. Reaction scheme for the synthesis of isoxazoles.

Similarly, other substituted isoxazoles have been prepared. All the synthesized compounds were re crystallized prior to use.

Spectral analysis: The structure of all the synthesized compounds was confirmed by spectroscopic techniques.

The IR spectra were recorded on Shimadzu FT-IR-8400 instrument using KBr pellet method. The Mass spectra were recorded on Shimadzu GC-MS-QP-2010 model using direct inlet probe technique. ${ }^{1} \mathrm{H}$ NMR was determined in DMSO solution on a Bruker Ac $400 \mathrm{MHz}$ spectrometer.

Antibacterial studies: The antibacterial activities of all synthesized compounds were studied in DMF and DMSO. Both the solvents were fractionally distilled by standard method [35].

In the present work, the antibacterial activities of the synthesized compounds have been screened against some Gram positive and Gram negative bacterial stains using agar well diffusion method.

Preparation of test compounds: The solutions were prepared at a concentration of $1 \mathrm{mg} / \mu \mathrm{l}$ for all the compounds.

Preparation of the plates and microbiological assay: The antibacterial evaluation was done by agar well diffusion method [36, 37] using Mueller Hinton Agar No.2 as the nutrient medium. The agar well diffusion method was preferred to be used in this study because it was found to be better than the disc diffusion method as suggested by Parekh et al. [37]. The bacterial strains were activated by inoculating a loop full of test strain in $25 \mathrm{ml}$ of N-broth and the same was incubated for $24 \mathrm{~h}$ in an incubator at $37^{\circ}$ C. $0.2 \mathrm{ml}$ of the activated strain was inoculated in Mueller Hinton Agar. Mueller Hinton Agar kept at $45^{\circ} \mathrm{C}$ was then poured in the Petri dishes and allowed to solidify. After solidification of the media, $0.85 \mathrm{~cm}$ ditch was made in the plates using a sterile cork borer and these were completely filled with the test solution. The plates were incubated for $24 \mathrm{~h}$ at $37^{\circ} \mathrm{C}$. The mean value obtained for the three wells was used to calculate the zone of growth inhibition of each sample. The controls were maintained for each bacterial strain and each solvent. The inhibition zone formed by these compounds against the particular test bacterial strain determined the antibacterial activities of these synthesized compounds.

\section{Results}

In both Cyanopyridine and Isoxazole series, ten compounds are synthesized. The synthesized compounds were purified through column using chloroform: methanol $(80: 20 \mathrm{v} / \mathrm{v})$. The purity of compounds was checked by TLC using appropriate solvent systems.

The physical parameters of synthesized cyanopyridine and isoxazole compounds are listed in Tables 1 and 2. 
Table 1. Physical constants of cyanopiridines.

\begin{tabular}{|c|c|c|c|c|c|}
\hline Sr. No. & $\begin{array}{l}\text { Compound } \\
\text { Code }\end{array}$ & $\mathbf{R}$ & $\begin{array}{c}\mathbf{R}_{\mathbf{f}}^{*} \\
\text { Value }\end{array}$ & $\stackrel{\text { M.P. }}{{ }^{\mathbf{0}} \mathrm{C}}$ & $\begin{array}{c}\text { Yield } \\
\%\end{array}$ \\
\hline 1 & NCP-1 & $4-\mathrm{OCH}_{3}-\mathrm{C}_{6} \mathrm{H}_{4}-$ & 0.65 & 212 & 69 \\
\hline 2 & NCP-2 & $4-\mathrm{NH}_{2}-\mathrm{C}_{6} \mathrm{H}_{4-}$ & 0.51 & 210 & 72 \\
\hline 3 & NCP-3 & $4-\mathrm{Br}-\mathrm{C}_{6} \mathrm{H}_{4-}$ & 0.49 & 225 & 75 \\
\hline 4 & NCP-4 & $4-\mathrm{NO}_{2}-\mathrm{C}_{6} \mathrm{H}_{4}-$ & 0.75 & 196 & 71 \\
\hline 5 & NCP-5 & $3-\mathrm{NO}_{2}-\mathrm{C}_{6} \mathrm{H}_{4}-$ & 0.69 & 178 & 63 \\
\hline 6 & NCP-6 & $4-\mathrm{OH}-\mathrm{C}_{6} \mathrm{H}_{4-}$ & 0.73 & 188 & 67 \\
\hline 7 & NCP-7 & $4-\mathrm{CH}_{3}-\mathrm{C}_{6} \mathrm{H}_{4}-$ & 0.52 & 162 & 64 \\
\hline 8 & NCP-8 & 4-Cl- $\mathrm{C}_{6} \mathrm{H}_{4-}$ & 0.58 & 154 & 75 \\
\hline 9 & NCP-9 & $2-\mathrm{OH}-\mathrm{C}_{6} \mathrm{H}_{4}-$ & 0.47 & 202 & 79 \\
\hline 10 & NCP-10 & $\mathrm{C}_{6} \mathrm{H}_{5^{-}}$ & 0.45 & 187 & 81 \\
\hline
\end{tabular}

Table 2. Physical constants of isoxazoles.

\begin{tabular}{|c|c|c|c|c|c|}
\hline Sr. No. & $\begin{array}{c}\text { Compound } \\
\text { Code }\end{array}$ & $\mathbf{R}$ & $\begin{array}{c}\mathbf{R}_{\mathbf{f}}^{*} \\
\text { Value }\end{array}$ & $\underset{{ }^{\mathbf{M}} \mathrm{C} . P .}{ }$ & $\begin{array}{c}\text { Yield } \\
\%\end{array}$ \\
\hline 1 & NISO-1 & $4-\mathrm{OCH}_{3}-\mathrm{C}_{6} \mathrm{H}_{4}-$ & 0.57 & 171 & 71 \\
\hline 2 & NISO-2 & $4-\mathrm{NH}_{2}-\mathrm{C}_{6} \mathrm{H}_{4-}$ & 0.68 & 184 & 65 \\
\hline 3 & NISO-3 & $4-\mathrm{Br}-\mathrm{C}_{6} \mathrm{H}_{4-}$ & 0.65 & 210 & 69 \\
\hline 4 & NISO-4 & $4-\mathrm{NO}_{2}-\mathrm{C}_{6} \mathrm{H}_{4-}$ & 0.61 & 168 & 72 \\
\hline 5 & NISO-5 & $3-\mathrm{NO}_{2}-\mathrm{C}_{6} \mathrm{H}_{4}-$ & 0.72 & 241 & 75 \\
\hline 6 & NISO-6 & $4-\mathrm{OH}-\mathrm{C}_{6} \mathrm{H}_{4}-$ & 0.75 & 222 & 64 \\
\hline 7 & NISO-7 & $4-\mathrm{CH}_{3}-\mathrm{C}_{6} \mathrm{H}_{4}-$ & 0.53 & 198 & 61 \\
\hline 8 & NISO-8 & $4-\mathrm{Cl}-\mathrm{C}_{6} \mathrm{H}_{4-}$ & 0.68 & 212 & 64 \\
\hline 9 & NISO-9 & $2-\mathrm{OH}-\mathrm{C}_{6} \mathrm{H}_{4}-$ & 0.71 & 202 & 67 \\
\hline 10 & NISO-10 & $\mathrm{C}_{6} \mathrm{H}_{5^{-}}$ & 0.78 & 235 & 58 \\
\hline
\end{tabular}

*Ethyl acetate: Hexane: 2:8

\section{Spectral data}

\section{Cyanopyridines:}

NCP-1 IR (cm ${ }^{-1}, \mathbf{K B r}$ ): 2933.83 (asym. C-H str.), 2841.24 (sym. C-H str.), 1456.30 (asym. C-H def.), 1363.72 (sym. C-H def.), 3061.13 (sym. aromatic C-H str.), 1508.38 (aromatic $\mathrm{C}=\mathrm{C}$ str.), 1658.84 (C=N str.), 1247.99 (C-N str.), 2208.57 (nitrile C-N str.), 3323.46 (N-H str.), 1600.97 (N-H def.), 1220.98 (asym. C-O-C str.), 1026.16 (sym. C-O-C str.), 1174.69 (C-F str.), 661.61 (C-Cl str.), ${ }^{1} \boldsymbol{H}$ NMR (DMSO-d $\left.{ }_{6}\right) \boldsymbol{\delta}(\mathbf{p p m}): 3.92$ (3H, singlet, $\left.-\mathrm{OCH}_{3}\right), 7.09-7.12$ (2H, doublet, Ar-CH), 7.50$7.54(1 \mathrm{H}$, triplet, Ar-CH), 7.66-7.69 (1H, doublet, Ar-CH), 7.75-7.77 (1H, doublet, Ar-CH), 7.84$8.11(4 \mathrm{H}$, multiplet, Ar-CH), $\boldsymbol{M S}:(\boldsymbol{m} / \mathbf{z})=404.8$

NCP-2 IR (cm ${ }^{-1}, \mathbf{K B r}$ ): 2933.22 (asym. C-H str.), 2841.28 (sym. C-H str.), 1456.10 (asym. C-H def.), 1363.60 (sym. C-H def.), 3061.17 (sym. aromatic C-H str.), 1521.04 (aromatic $\mathrm{C}=\mathrm{C}$ str.), 1658.42 (C=N str.), 1247.41(C-N str.), 2232.14 (nitrile C-N str.), 3368.81 (N-H str.), 1600.93 (N-H def.), 1220.91 (asym. C-O-C str.), 1026.10(sym. C-O-C str.), 1174.61 (C-F str.), 721.40 (C-Cl str.), ${ }^{1} \boldsymbol{H}$ NMR (DMSO-d 6 ) $\delta($ ppm): 7.04-7.10 (2H, doublet, Ar-CH), 7.51-7.53 (1H, triplet, Ar-CH), 7.65-7.67 (1H, doublet, Ar-CH), 7.74-7.76 (1H, doublet, Ar-CH), 7.85-8.10 (4H, multiplet, Ar-CH), $7.93\left(2 \mathrm{H}\right.$, singlet, $\left.-\mathrm{NH}_{2}\right), \boldsymbol{M S}:(\boldsymbol{m} / \boldsymbol{z})=389.8$

NCP-3 IR (cm $\left.{ }^{-1}, \mathbf{K B r}\right): 2933.81$ (asym. C-H str.), 2841.35 (sym. C-H str.), 1456.12 (asym. C-H def.), 1363.42 (sym. C-H def.), 3061.14 (sym. aromatic C-H str.), 1508.35 (aromatic $\mathrm{C}=\mathrm{C}$ str.), 1658.81 (C=N str.), 1247.87 (C-N str.), 2208.51 (nitrile C-N str.), 3344.22 (N-H str.), 1600.55 (N-H def.), 1220.32 (asym. C-O-C str.), 1026.19 (sym. C-O-C str.), 1174.64 (C-F str.), 712.49 (C-Cl str.), 
${ }^{1} \boldsymbol{H}$ NMR (DMSO-d $)_{6} \boldsymbol{\delta}(\mathbf{p p m})$ : 7.06-7.10 (2H, doublet, Ar-CH), 7.52-7.53 (1H, triplet, Ar-CH), 7.62-7.65 (1H, doublet, Ar-CH), 7.73-7.76 (1H, doublet, Ar-CH), 7.82-8.05 (4H, multiplet, Ar-CH), MS: $(\boldsymbol{m} / \mathbf{z})=453.7$

NCP-4 IR (cm $\left.{ }^{-1}, \mathbf{K B r}\right): 2933.66$ (asym. C-H str.), 2841.34 (sym. C-H str.), 1456.27 (asym. C-H def.), 1363.98 (sym. C-H def.), 3061.47 (sym. aromatic C-H str.), 1508.75 (aromatic $\mathrm{C}=\mathrm{C}$ str.), 1658.74 (C=N str.), 1247.94 (C-N str.), 2221.74 (nitrile C-N str.), 3323.27 (N-H str.), 1600.77 (N-H def.), 1220.64 (asym. C-O-C str.), 1026.04 (sym. C-O-C str.), 1174.41 (C-F str.), 724.33 (C-Cl str.), ${ }^{1} \boldsymbol{H}$ NMR (DMSO-d 6 ) $\boldsymbol{\delta}(\mathbf{p p m})$ : 7.04-7.09 (2H, doublet, Ar-CH), 7.52-7.58 (1H, triplet, Ar-CH), 7.63-7.68, (1H, doublet, Ar-CH), 7.72-7.75 (1H, doublet, Ar-CH), 7.80-8.09 (4H, multiplet, Ar$\mathrm{CH}), \boldsymbol{M S}:(\boldsymbol{m} / \mathbf{z})=419.8$

NCP-5 IR (cm $\left.{ }^{-1}, \boldsymbol{K B r}\right): 2933.72$ (asym. C-H str.), 2841.37 (sym. C-H str.), 1456.74 (asym. C-H def.), 1363.87 (sym. C-H def.), 3061.41(sym. aromatic C-H str.), 1508.55 (aromatic $\mathrm{C}=\mathrm{C}$ str.), 1658.44 (C=N str.), 1247.77 (C-N str.), 2234.37 (nitrile C-N str.), 3315.57 (N-H str.), 1600.97 (N-H def.), 1220.98 (asym. C-O-C str.), 1026.54 (sym. C-O-C str.), 1174.27 (C-F str.), 668.32 (C-Cl str.), ${ }^{1} \boldsymbol{H}$ NMR (DMSO-d 6 ) $\delta($ ppm): 7.07-7.10 (2H, doublet, Ar-CH), 7.50-7.54 (1H, triplet, Ar-CH), 7.66-7.69 (1H, doublet, Ar-CH), 7.75-7.77 (1H, doublet, Ar-CH), 7.84-8.11 (3H, multiplet, Ar-CH), $8.26(1 \mathrm{H}$, singlet, Ar-CH), MS: $(\mathbf{m} / z)=419.8$

NCP-6 IR (cm $\left.{ }^{-1}, \boldsymbol{K B r}\right): 2933.77$ (asym. C-H str.), 2841.47 (sym. C-H str.), 1456.64 (asym. C-H def.), 1363.34 (sym. C-H def.), 3061.24 (sym. aromatic C-H str.), 1508.79 (aromatic $\mathrm{C}=\mathrm{C}$ str.), 1658.65 (C=N str.), 1247.93 (C-N str.), 2232.24 (nitrile C-N str.), 3298.77 (N-H str.), 1600.54 (N-H def.), 1220.47 (asym. C-O-C str.), 1026.33 (sym. C-O-C str.), 1174.47 (C-F str.), 668.34 (C-Cl str.), ${ }^{1} \boldsymbol{H}$ NMR (DMSO-d 6 ) $\delta($ ppm): 7.02-7.10 (2H, doublet, Ar-CH), 7.55-7.57 (1H, triplet, Ar-CH), 7.67-7.69 (1H, doublet, Ar-CH), 7.76-7.78 (1H, doublet, Ar-CH), 7.86-8.13 (4H, multiplet, Ar-CH), $9.12(1 \mathrm{H}$, singlet, $-\mathrm{OH}), \boldsymbol{M S}:(\boldsymbol{m} / \mathbf{z})=390.8$

NCP-7 IR (cm $\left.{ }^{-1}, \mathbf{K B r}\right): 2933.43$ (asym. C-H str.), 2841.57 (sym. C-H str.), 1456.77 (asym. C-H def.), 1363.78 (sym. C-H def.), 3061.17 (sym. aromatic $\mathrm{C}-\mathrm{H}$ str.), 1508.64 (aromatic $\mathrm{C}=\mathrm{C}$ str.), 1658.72 (C=N str.), 1247.87 (C-N str.), 2208.38 (nitrile C-N str.), 3275.87 (N-H str.), 1600.54 (N-H def.), 1220.91 (asym. C-O-C str.), 1026.24 (sym. C-O-C str.), 1174.21 (C-F str.), 678.41 (C-Cl str.), ${ }^{1} \boldsymbol{H}$ NMR (DMSO-d $\left.{ }_{6}\right) \boldsymbol{\delta}(\mathbf{p p m}): 1.25$ (3H, singlet, $\left.-\mathrm{CH}_{3}\right), 7.09-7.12$ (2H, doublet, Ar-CH), 7.50-7.54 $(1 \mathrm{H}$, triplet, Ar-CH), 7.66-7.69 $(1 \mathrm{H}$, doublet, Ar-CH), 7.75-7.77 $(1 \mathrm{H}$, doublet, Ar-CH), 7.84-8.11 (4H, multiplet, Ar-CH), MS: $(\boldsymbol{m} / z)=388.8$

NCP-8 IR (cm $\left.{ }^{-1}, \mathbf{K B r}\right): 2933.66$ (asym. C-H str.), 2841.34 (sym. C-H str.), 1456.55 (asym. C-H def.), 1363.67 (sym. C-H def.), 3061.23 (sym. aromatic C-H str.), 1508.47 (aromatic $\mathrm{C}=\mathrm{C}$ str.), 1658.76 (C=N str.), 1247.94 (C-N str.), 2208.24 (nitrile C-N str.), 3304.42 (N-H str.), 1600.46 (N-H def.), 1220.98 (asym. C-O-C str.), 1026.37 (sym. C-O-C str.), 1174.77 (C-F str.), 731.64 (C-Cl str.), ${ }^{1} \boldsymbol{H}$ NMR (DMSO-d $\left.)_{6}\right) \boldsymbol{\delta}(\mathbf{p p m}):$ 7.04-7.10 (2H, doublet, Ar-CH), 7.51-7.55 (1H, triplet, Ar-CH), 7.62-7.65 (1H, doublet, Ar-CH), 7.73-7.76 (1H, doublet, Ar- $\mathrm{CH}), 7.81-8.05(4 \mathrm{H}$, multiplet, Ar-CH), MS: $(\boldsymbol{m} / \mathbf{z})=409.2$

NCP-9 IR (cm $\left.{ }^{-1}, \boldsymbol{K B r}\right): 2933.47$ (asym. C-H str.), 2841.75 (sym. C-H str.), 1456.11 (asym. C-H def.), 1363.52 (sym. C-H def.), 3061.44 (sym. aromatic C-H str.), 1508.78 (aromatic $\mathrm{C}=\mathrm{C}$ str.), 1658.74 (C=N str.), 1247.49 (C-N str.), 2204.32 (nitrile C-N str.), 3323.78 (N-H str.), 1600.87 (N-H def.), 1220.85 (asym. C-O-C str.), 1026.17 (sym. C-O-C str.), 1174.60 (C-F str.), 711.85 (C-Cl str.), ${ }^{1} \boldsymbol{H}$ NMR (DMSO-d $)_{6} \boldsymbol{\delta}(\mathbf{p p m}): 7.25-7.29$ (2H, doublet, Ar-CH), 7.50-7.54 (1H, triplet, Ar-CH), 7.66-7.69 (1H, doublet, Ar-CH), 7.75-7.77 (1H, doublet, Ar-CH), 7.84-8.11 (4H, multiplet, Ar-CH), $9.18(1 \mathrm{H}$, singlet, $-\mathrm{OH}), \mathbf{M S}:(\boldsymbol{m} / \mathbf{z})=409.2$

NCP-10 IR (cm $\left.{ }^{-1}, \mathbf{K B r}\right): 2933.41$ (asym. C-H str.), 2841.24 (sym. C-H str.), 1456.75 (asym. C-H def.), 1363.46 (sym. C-H def.), 3061.61 (sym. aromatic $\mathrm{C}-\mathrm{H}$ str.), 1508.24 (aromatic $\mathrm{C}=\mathrm{C}$ str.), 1658.42 (C=N str.), 1247.87 (C-N str.), 2234.58 (nitrile C-N str.), 3285.38 (N-H str.), 1600.84 (N-H def.), 1220.79 (asym. C-O-C str.), 1026.54 (sym. C-O-C str.), 1174.85 (C-F str.), 715.58 (C-Cl str.), 
${ }^{1} \boldsymbol{H}$ NMR (DMSO-d $)$ $\delta(\mathbf{p p m}):$ 7.33-7.36 (2H, doublet, Ar-CH), 7.50-7.54 (1H, triplet, Ar-CH), 7.66-7.69 (1H, doublet, Ar-CH), 7.75-7.77 (1H, doublet, Ar-CH), 7.84-8.11 (4H, multiplet, Ar-CH), MS: $(\boldsymbol{m} / \mathbf{z})=374.8$

\section{Isoxazoles}

NISO-1 IR (cm ${ }^{-1}, \boldsymbol{K B r}$ ): 2899.11 (asym. C-H str.), 2845.10 (sym. C-H str.), 1456.30 (asym. C-H def.), 1423.51 (sym. C-H def.), 3020.63 (sym. aromatic $\mathrm{C}-\mathrm{H}$ str.), 1506.46 (aromatic $\mathrm{C}=\mathrm{C}$ str.), 1664.62 ( $\mathrm{C}=\mathrm{N}$ str.), 1300.07 (C-N str.), 833.28 (N-O str.), 1247.99 (asym. C-O-C str.), 1026.16 (sym. C-O-C str.), 1182.40 (C-F str.), 725.26 (C-Cl str.), ${ }^{1} \boldsymbol{H}$ NMR (DMSO-d ( $_{\boldsymbol{6}} \boldsymbol{\delta}$ (ppm): 3.79 (3H, singlet, $\left.-\mathrm{OCH}_{3}\right), 6.86-6.88(2 \mathrm{H}$, doublet, Ar-CH), $7.13(1 \mathrm{H}$, singlet, Ar-CH), 7.33-7.35 $(2 \mathrm{H}$, doublet, Ar-CH), 7.44-7.48 $(1 \mathrm{H}$, triplet, Ar-CH), 7.69-7.71 $(1 \mathrm{H}$, doublet, Ar-CH), 7.85-7.87 $(1 \mathrm{H}$, doublet, Ar-CH), $8.42(1 \mathrm{H}$, singlet, Ar-CH), $M S:(\boldsymbol{m} / z)=354.8$

NISO-2 IR (cm ${ }^{-1}, \boldsymbol{K B r}$ ): 2899.25 (asym. C-H str.), 2845.65 (sym. C-H str.), 1456.84 (asym. C-H def.), 1423.34 (sym. C-H def.), 3020.24 (sym. aromatic 0C-H str.), 1488.52 (aromatic $\mathrm{C}=\mathrm{C}$ str.), 1664.42 (C=N str.), 1311.89 (C-N str.), 824.49 (N-O str.), 1247.80 (asym. C-O-C str.), 1026.27 (sym. C-O-C str.), 1182.34 (C-F str.), 741.20 (C-Cl str.), ${ }^{1} \boldsymbol{H}$ NMR (DMSO-d ( $_{\text {) }} \boldsymbol{\delta}$ (ppm): 6.82-6.86 $(2 \mathrm{H}$, doublet, Ar-CH), $7.12(1 \mathrm{H}$, singlet, Ar-CH), 7.31-7.34 $(2 \mathrm{H}$, doublet, Ar-CH), 7.45-7.49 $(1 \mathrm{H}$, triplet, Ar-CH), 7.68-7.70 (1H, doublet, Ar-CH), 7.80-7.83 $(1 \mathrm{H}$, doublet, Ar-CH), $8.40(1 \mathrm{H}$, singlet, Ar-CH), $7.90\left(2 \mathrm{H}\right.$, singlet, $\left.-\mathrm{NH}_{2}\right), \boldsymbol{M S}:(\boldsymbol{m} / \mathbf{z})=339.8$

NISO-3 IR ( $\mathbf{c m}^{-1}, \mathbf{K B r}$ ): 2899.54 (asym. C-H str.), 2845.63 (sym. C-H str.), 1456.26 (asym. C-H def.), 1423.84 (sym. C-H def.), 3020.55 (sym. aromatic C-H str.), 1524.38 (aromatic $\mathrm{C}=\mathrm{C}$ str.), 1664.38 (C=N str.), 1278.21 (C-N str.), 828.71 (N-O str.), 1247.05 (asym. C-O-C str.), 1026.04 (sym. C-O-C str.), 1182.36 (C-F str.), 725.84 (C-Cl str.), ${ }^{1} \boldsymbol{H}$ NMR (DMSO-d ( $_{\text {) }} \boldsymbol{\delta}$ (ppm): 6.81-6.83 $(2 \mathrm{H}$, doublet, Ar-CH), $7.10(1 \mathrm{H}$, singlet, Ar- $\mathrm{CH}), 7.32-7.36(2 \mathrm{H}$, doublet, Ar- $\mathrm{CH}), 7.46-7.49(1 \mathrm{H}$, triplet, Ar-CH), 7.70-7.73 (1H, doublet, Ar-CH), 7.83-7.85 (1H, doublet, Ar-CH), $8.40(1 \mathrm{H}$, singlet, Ar-CH), MS: $(m / z)=403.6$

NISO-4 IR (cm ${ }^{-1}, \boldsymbol{K B r}$ ): 2899.29 (asym. C-H str.), 2845.25 (sym. C-H str.), 1456.90 (asym. C-H def.), 1423.57 (sym. C-H def.), 3020.55 (sym. aromatic $\mathrm{C}-\mathrm{H}$ str.), 1498.63 (aromatic $\mathrm{C}=\mathrm{C}$ str.), 1664.24 (C=N str.), 1247.29 (C-N str.), 833.28 (N-O str.), 1247.57 (asym. C-O-C str.), 1026.27 (sym. C-O-C str.), 1182.34 (C-F str.), 712.79 (C-Cl str.), ${ }^{1} \boldsymbol{H}$ NMR (DMSO-d $)$ o(ppm): 6.82-6.84 (2H, doublet, Ar-CH), $7.14(1 \mathrm{H}$, singlet, Ar-CH), 7.31-7.33 (2H, doublet, Ar-CH), 7.41-7.44 $(1 \mathrm{H}$, triplet, Ar-CH), 7.60-7.65 (1H, doublet, Ar-CH), 7.80-7.83 (1H, doublet, Ar-CH), 8.39 (1H, singlet, Ar-CH), MS: $(\boldsymbol{m} / z)=369.7$

NISO-5 IR (cm ${ }^{-1}, \boldsymbol{K B r}$ ): 2899.14 (asym. C-H str.), 2845.37 (sym. C-H str.), 1456.88 (asym. C-H def.), 1423.71 (sym. C-H def.), 3020.44 (sym. aromatic $\mathrm{C}-\mathrm{H}$ str.), 1508.64 (aromatic $\mathrm{C}=\mathrm{C}$ str.), 1664.62 (C=N str.), 1325.54 (C-N str.), 812.62 (N-O str.), 1247.79 (asym. C-O-C str.), 1026.10 (sym. C-O-C str.), 1182.46 (C-F str.), 724.41 (C-Cl str.), ${ }^{1} \boldsymbol{H}$ NMR (DMSO-d ( $_{\text {) }} \boldsymbol{\delta}$ (ppm): 6.89 (1H, siglet, Ar-CH), 7.29-7.31 (2H, doublet, Ar-CH), 7.43-7.45 (1H, multiplet, Ar-CH), 7.44-7.48 (1H, triplet, Ar-CH), 7.69-7.71 (1H, doublet, Ar-CH), 7.85-7.87 (1H, doublet, Ar-CH), $8.42(1 \mathrm{H}$, singlet, Ar-CH), MS: $(\boldsymbol{m} / z)=369.7$

NISO-6 IR (cm ${ }^{-1}, \boldsymbol{K B r}$ ): 2899.25 (asym. C-H str.), 2845.55 (sym. C-H str.), 1456.27 (asym. C-H def.), 1423.33 (sym. C-H def.), 3020.78 (sym. aromatic C-H str.), 1522.65 (aromatic $\mathrm{C}=\mathrm{C}$ str.), 1664.42 (C=N str.), 1300.07 (C-N str.), 812.62 (N-O str.), 1247.97 (asym. C-O-C str.), 1026.26 (sym. C-O-C str.), 1182.79 (C-F str.), 745.35 (C-Cl str.), ${ }^{1} \boldsymbol{H}$ NMR (DMSO-d ( $_{6}$ o(ppm): 6.84-6.86 $(2 \mathrm{H}$, doublet, Ar-CH), $7.11(1 \mathrm{H}$, singlet, Ar- $\mathrm{CH}), 7.34-7.36(2 \mathrm{H}$, doublet, Ar- $\mathrm{CH}), 7.45-7.47(1 \mathrm{H}$, triplet, Ar-CH), 7.70-7.72 (1H, doublet, Ar-CH), 7.84-7.86 (1H, doublet, Ar-CH), $8.40(1 \mathrm{H}$, singlet, Ar-CH), $9.10(1 \mathrm{H}$, singlet, $-\mathrm{OH}), \mathbf{M S}:(\boldsymbol{m} / \mathbf{z})=340.7$

NISO-7 IR (cm ${ }^{-1}, \boldsymbol{K B r}$ ): 2899.24 (asym. C-H str.), 2845.18 (sym. C-H str.), 1456.22 (asym. C-H def.), 1423.42 (sym. C-H def.), 3020.62 (sym. aromatic $\mathrm{C}-\mathrm{H}$ str.), 1528.54 (aromatic $\mathrm{C}=\mathrm{C}$ str.), 1664.22 ( $\mathrm{C}=\mathrm{N}$ str.), 1308.98 (C-N str.), 812.62 (N-O str.), 1247.67 (asym. C-O-C str.), 1026.17 
(sym. C-O-C str.), 1182.16 (C-F str.), 745.35 (C-Cl str.), ${ }^{1} \boldsymbol{H}$ NMR (DMSO-d 6 ) $\boldsymbol{\delta}$ (ppm): 1.21 (3H, singlet, $\left.-\mathrm{CH}_{3}\right), 6.81-6.84$ (2H, doublet, Ar- $\left.\mathrm{CH}\right), 7.18(1 \mathrm{H}$, singlet, Ar- $\mathrm{CH}), 7.30-7.33$ (2H, doublet, Ar- $\mathrm{CH}), 7.40-7.44(1 \mathrm{H}$, triplet, Ar- $\mathrm{CH}), 7.68-7.72(1 \mathrm{H}$, doublet, Ar- $\mathrm{CH}), 7.84-7.86(1 \mathrm{H}$, doublet, Ar-CH), $8.40(1 \mathrm{H}$, singlet, Ar-CH), MS: $(\boldsymbol{m} / z)=338.8$

NISO-8 IR (cm ${ }^{-1}, \boldsymbol{K B r}$ ): 2899.20 (asym. C-H str.), 2845.39 (sym. C-H str.), 1456.21 (asym. C-H def.), 1423.46 (sym. C-H def.), 3020.67 (sym. aromatic C-H str.), 1531.54 (aromatic $\mathrm{C}=\mathrm{C}$ str.), 1664.72 (C=N str.), 1333.61 (C-N str.), 833.18 (N-O str.), 1247.97 (asym. C-O-C str.), 1026.29

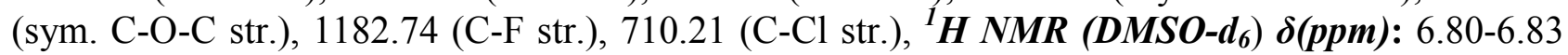
$(2 \mathrm{H}$, doublet, Ar-CH), $7.13(1 \mathrm{H}$, singlet, Ar- $\mathrm{CH}), 7.31-7.34(2 \mathrm{H}$, doublet, Ar-CH), 7.42-7.46 $(1 \mathrm{H}$, triplet, Ar-CH), 7.68-7.71 (1H, doublet, Ar-CH), 7.80-7.83 (1H, doublet, Ar-CH), 8.45 (1H, singlet, Ar-CH), MS: $(\boldsymbol{m} / z)=359.2$

NISO-9 IR ( $\boldsymbol{c m}^{-1}, \boldsymbol{K B r}$ ): 2899.24 (asym. C-H str.), 2845.17 (sym. C-H str.), 1456.54 (asym. C-H def.), 1423.71 (sym. C-H def.), 3020.53 (sym. aromatic $\mathrm{C}-\mathrm{H}$ str.), 1514.38 (aromatic $\mathrm{C}=\mathrm{C}$ str.), 1664.67 (C=N str.), 1298.75 (C-N str.), 841.21 (N-O str.), 1247.23 (asym. C-O-C str.), 1026.28 (sym. C-O-C str.), 1182.33 (C-F str.), 724.32 (C-Cl str.), ${ }^{1} \boldsymbol{H}$ NMR (DMSO-d $)$ o(ppm): 6.90-6.96 $(2 \mathrm{H}$, doublet, Ar-CH), 7.23-7.25 (2H, multiplet, Ar-CH), 7.43-7.45 (1H, triplet, Ar-CH), 7.68-7.70 $(1 \mathrm{H}$, doublet, Ar-CH), 7.87-7.89 (1H, doublet, Ar-CH), $8.46(1 \mathrm{H}$, singlet, Ar-CH), $9.17(1 \mathrm{H}$, singlet, $-\mathrm{OH}), \boldsymbol{M S}:(\boldsymbol{m} / \mathbf{z})=340.7$

NISO-10 IR (cm ${ }^{-1}, \boldsymbol{K B r}$ ): 2899.29 (asym. C-H str.), 2845.99 (sym. C-H str.), 1456.14 (asym. C-H def.), 1423.16 (sym. C-H def.), 3020.73 (sym. aromatic $\mathrm{C}-\mathrm{H}$ str.), 1501.52 (aromatic $\mathrm{C}=\mathrm{C}$ str.), 1664.56 (C=N str.), 1300.26 (C-N str.), 833.28 (N-O str.), 1247.57 (asym. C-O-C str.), 1026.46

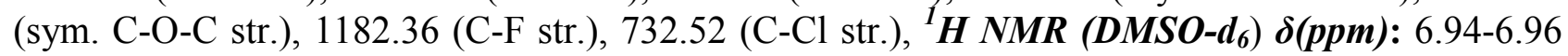
(2H, doublet, Ar-CH), 7.45-7.47 (3H, multiplet, Ar-CH), 7.44-7.48 (1H, triplet, Ar-CH), 7.62-7.65 $(1 \mathrm{H}$, doublet, Ar-CH), 7.83-7.85 $(1 \mathrm{H}$, doublet, Ar-CH), $8.44(1 \mathrm{H}$, singlet, Ar- $\mathrm{CH}), \boldsymbol{M S}:(\boldsymbol{m} / \mathbf{z})=$ 324.7

\section{Antibacterial activity}

\section{Cyanopyridines:}

Fig. 3 showed inhibition against Gram positive bacteria in DMSO. It is observed that against B. cereus except NCP-4, other compounds exhibited inhibition and is maximum for NCP-8 followed by NCP-6. There is no inhibition observed by any compounds against $M$. flavus and $S$. aureus.

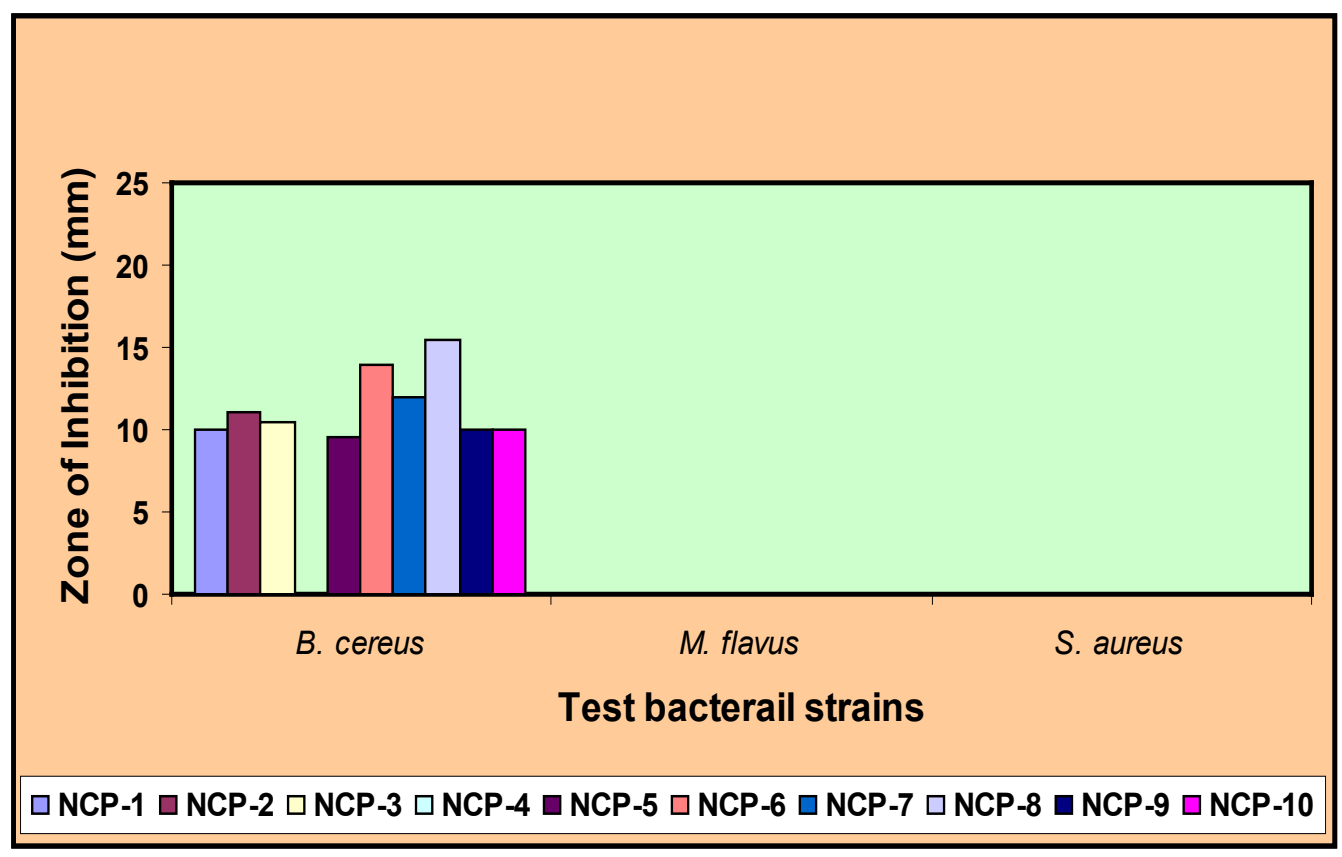

Figure 3. Antibacterial activity of cyanopyridines against Gram positive bacteria in DMSO. 
However, in DMF, all the compounds exhibited inhibition against B. cereus and $S$. aureus as shown in Fig. 4. Further, maximum activity is for NCP-8. Against M. flavus, only NCP-9 and NCP10 exhibited inhibition and inhibition is same for both the compounds.

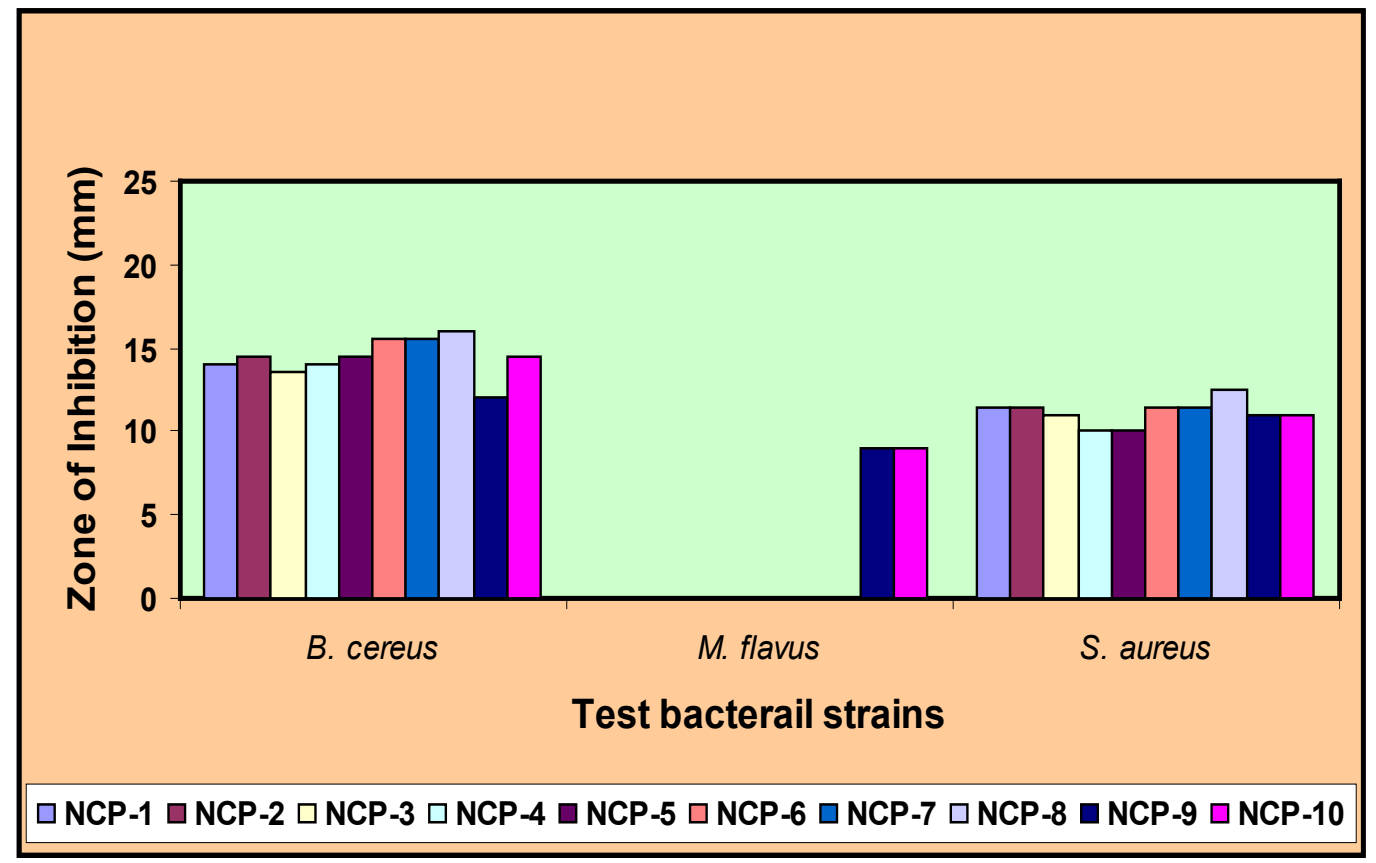

Figure 4. Antibacterial activity of cyanopyridines against Gram positive bacteria in DMF.

In case of Gram negative bacteria, Figs. 5 and 6 show zone of inhibition in DMSO and DMF respectively.

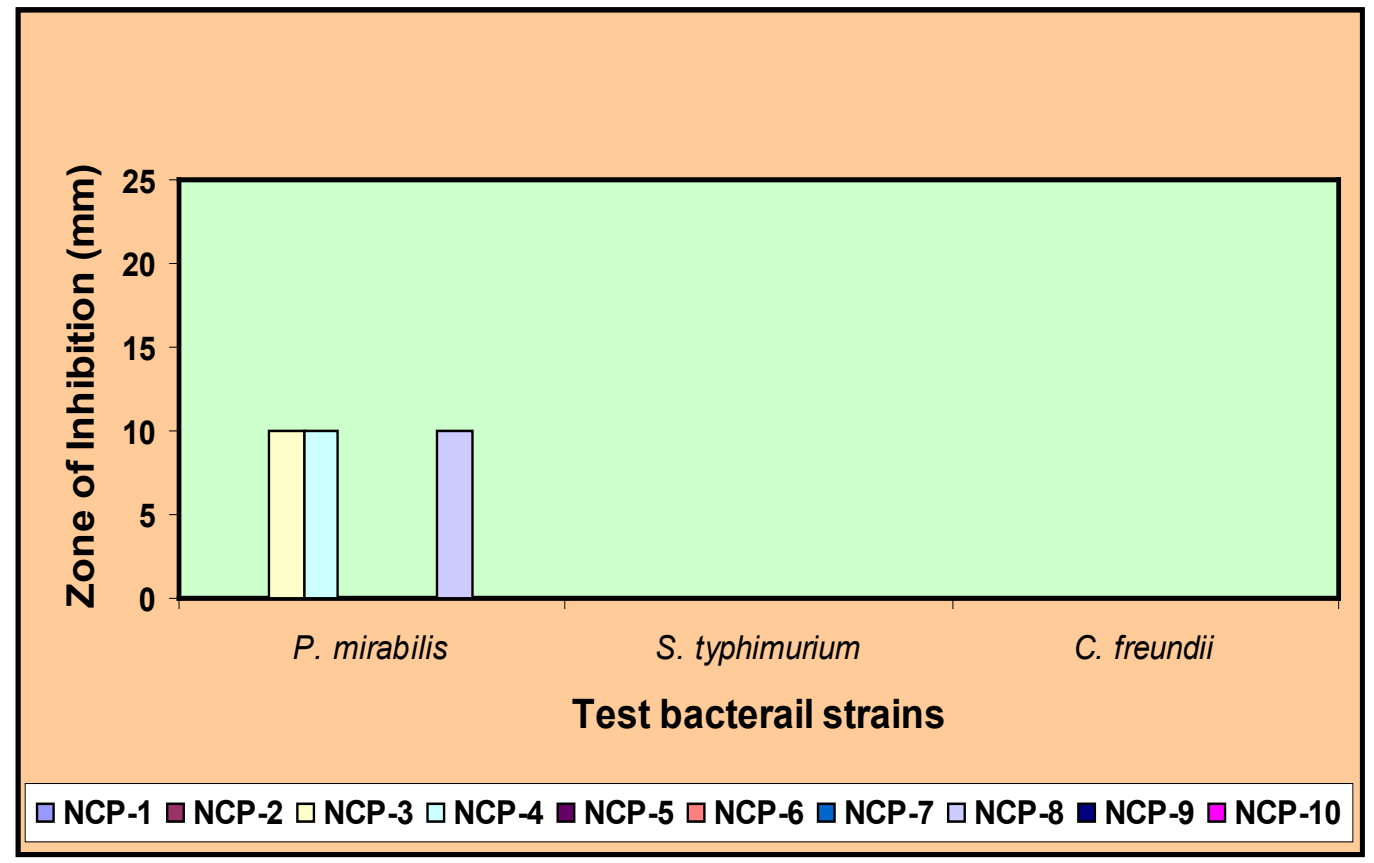

Figure 5. Antibacterial activity of cyanopyridines against Gram negative bacteria in DMSO. 


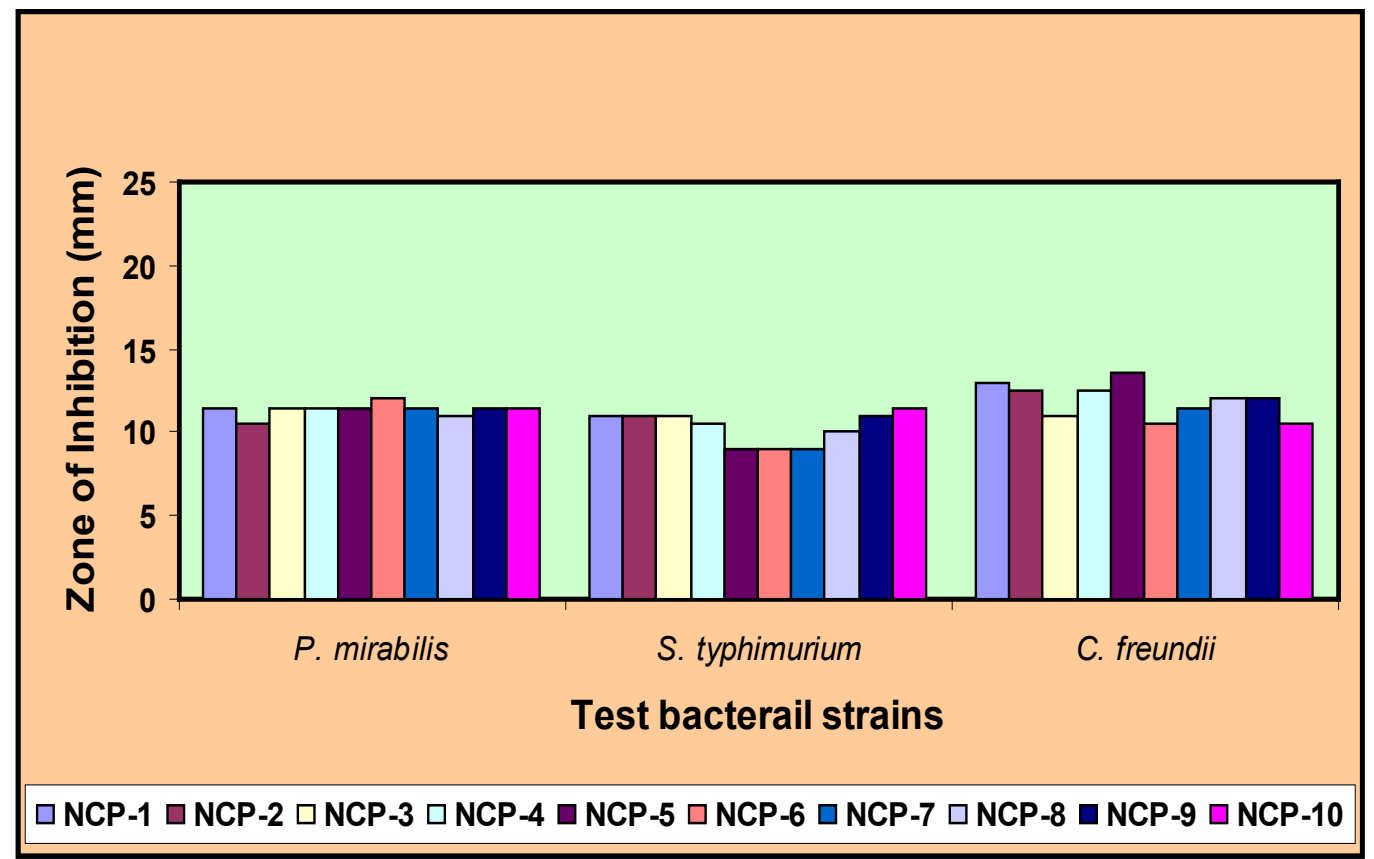

Figure 6. Antibacterial activity of cyanopyridines against Gram negative bacteria in DMF.

In DMSO, the studied compounds do not exhibit any activity against $S$. typhimurium and C. freundii. However, only NCP-3, NCP-4 and NCP-8 showed same inhibition for P. mirabilis. In DMF, all the compounds showed activity against all the three gram negative bacteria. For P. mirabilis, inhibition is almost similar for all the compounds. For $S$. typhimurium, maximum is for NCP-10 and minimum for NCP-5, NCP-6 and NCP-7.

\section{Isoxazoles}

Figs. 7 and 8 show inhibition against Gram positive bacteria in DMSO and DMF respectively.

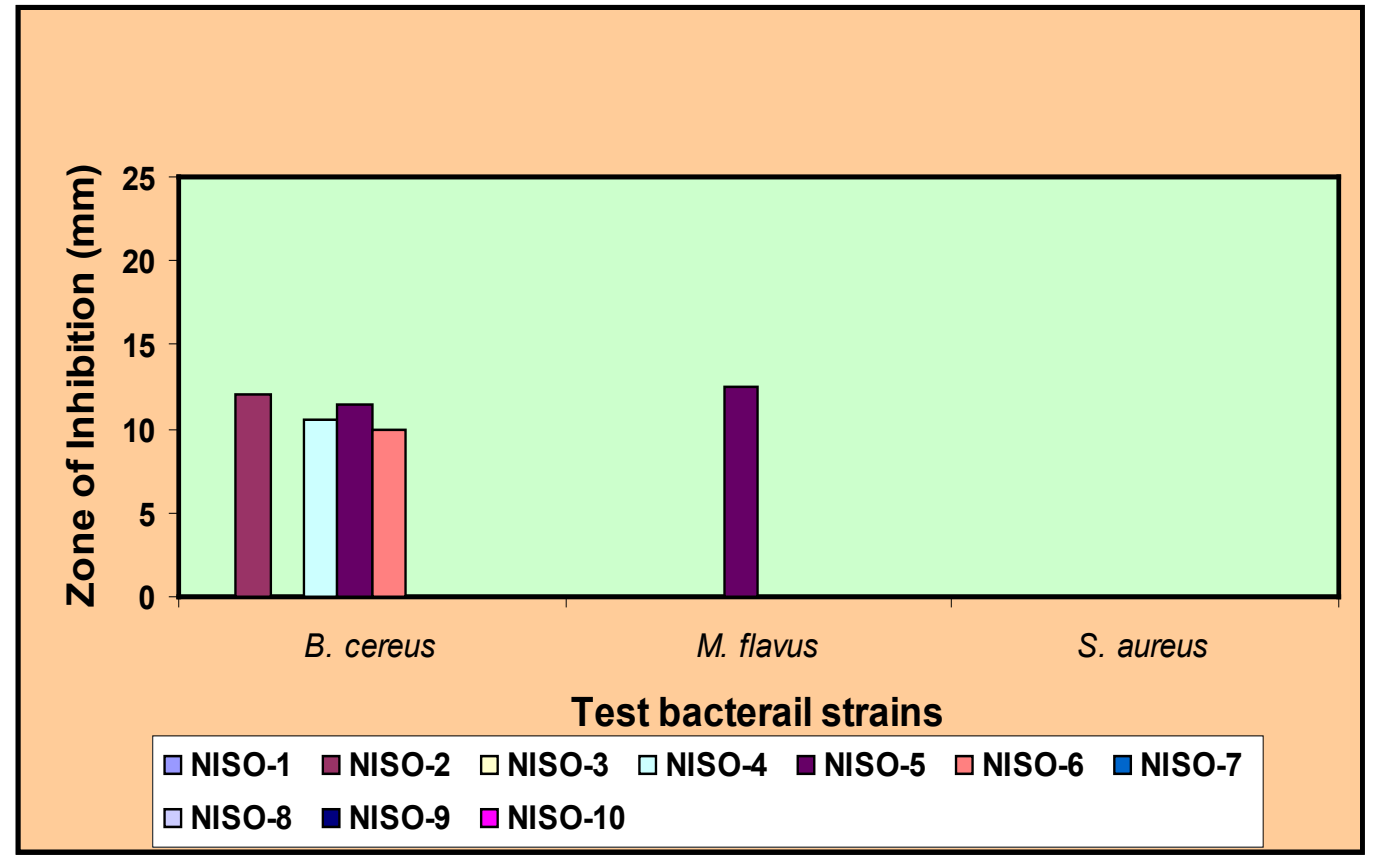

Figure 7. Antibacterial activity of isoxazoles against Gram positive bacteria in DMSO.

In DMSO, for B. cereus, only NISO-2, NISO-4, NISO-5 and NISO-6 showed inhibition. Other compounds had no effect at all. In DMF, against $B$. cereus, all compounds exhibited inhibition and maximum inhibition is for NISO-6. 


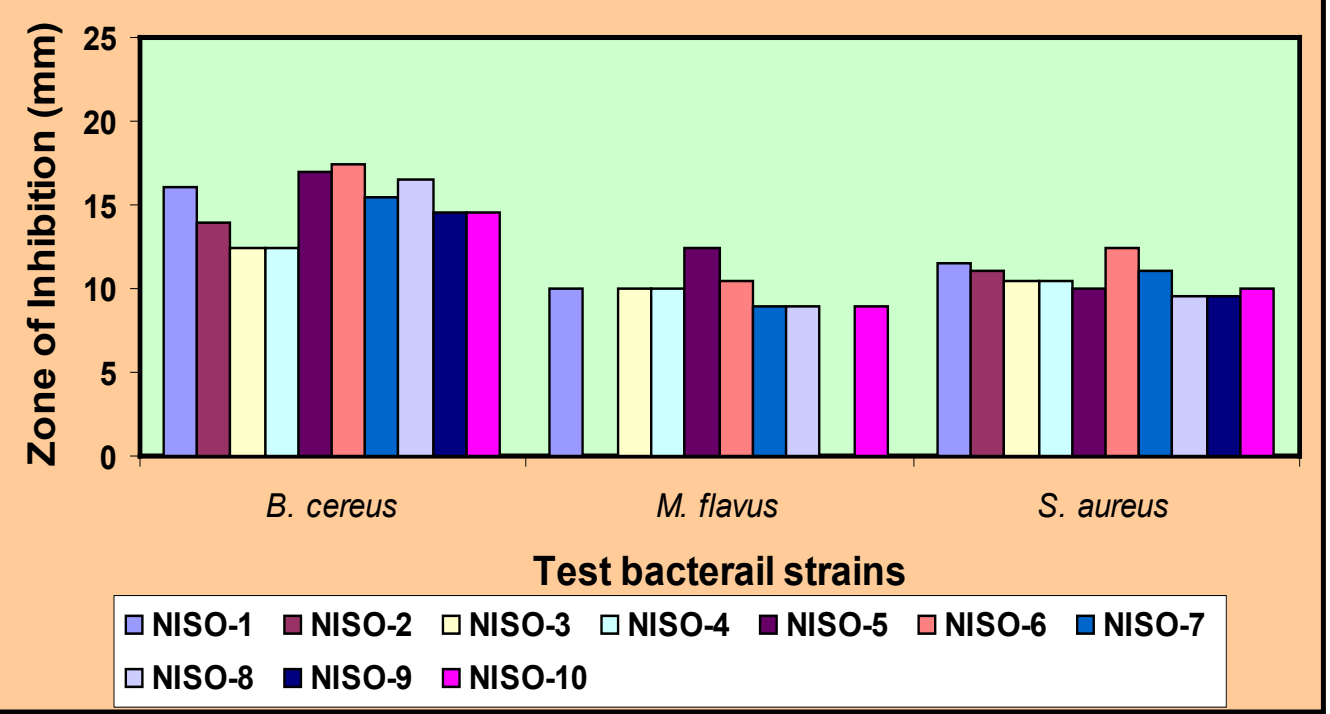

Figure 8. Antibacterial activity of isoxazoles against Gram positive bacteria in DMF.

Against Gram negative bacteria, zone of inhibition in DMSO and DMF are shown in Figs. 9 and 10 respectively.

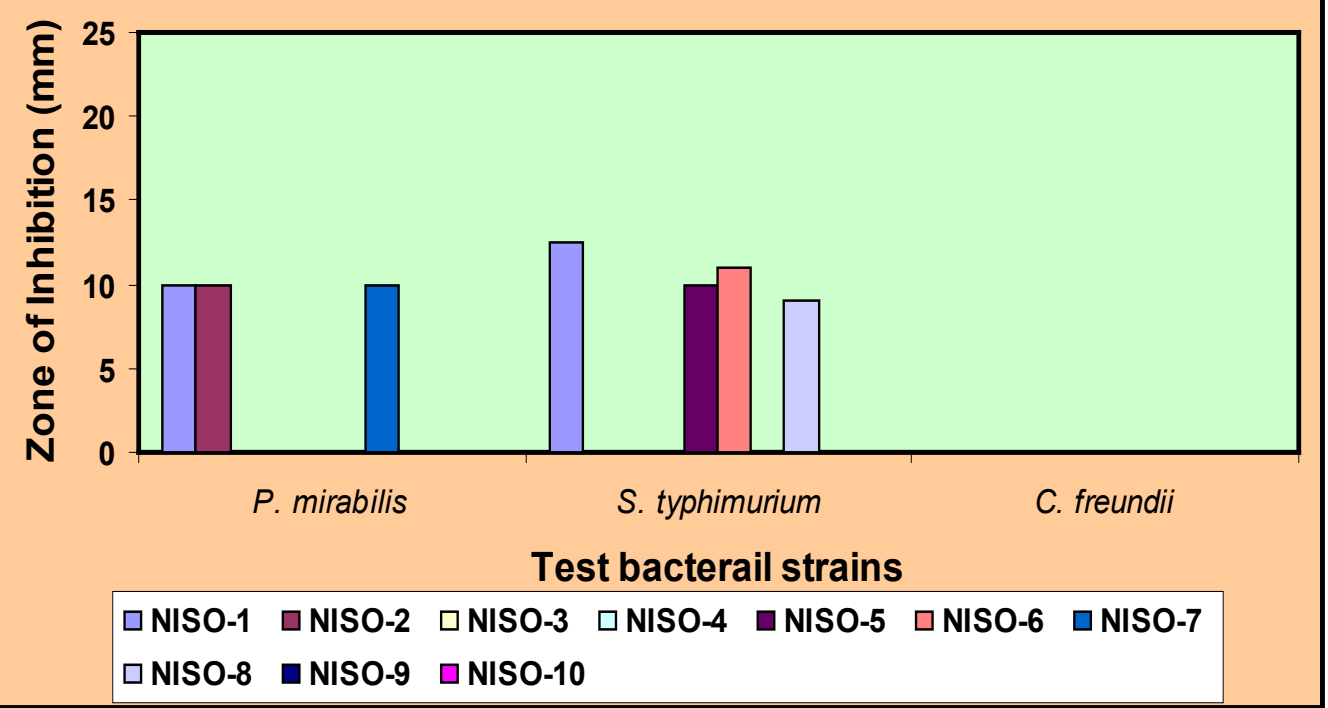

Figure 9. Antibacterial activity of isoxazoles against Gram negative bacteria in DMSO.

In DMSO, against $P$. mirabilis only NISO-1, NISO-2 and NISO-7 showed inhibition which is of same magnitude. Others compounds showed no inhibition at all. For $S$. typhimurium NISO-1, NISO-5, NISO-6 and NISO-8 showed inhibition. Other compounds had no effect on this bacterium. Maximum inhibition is for NISO-1 and minimum for NISO-8. No inhibition is observed against C. freundii by any compounds. 


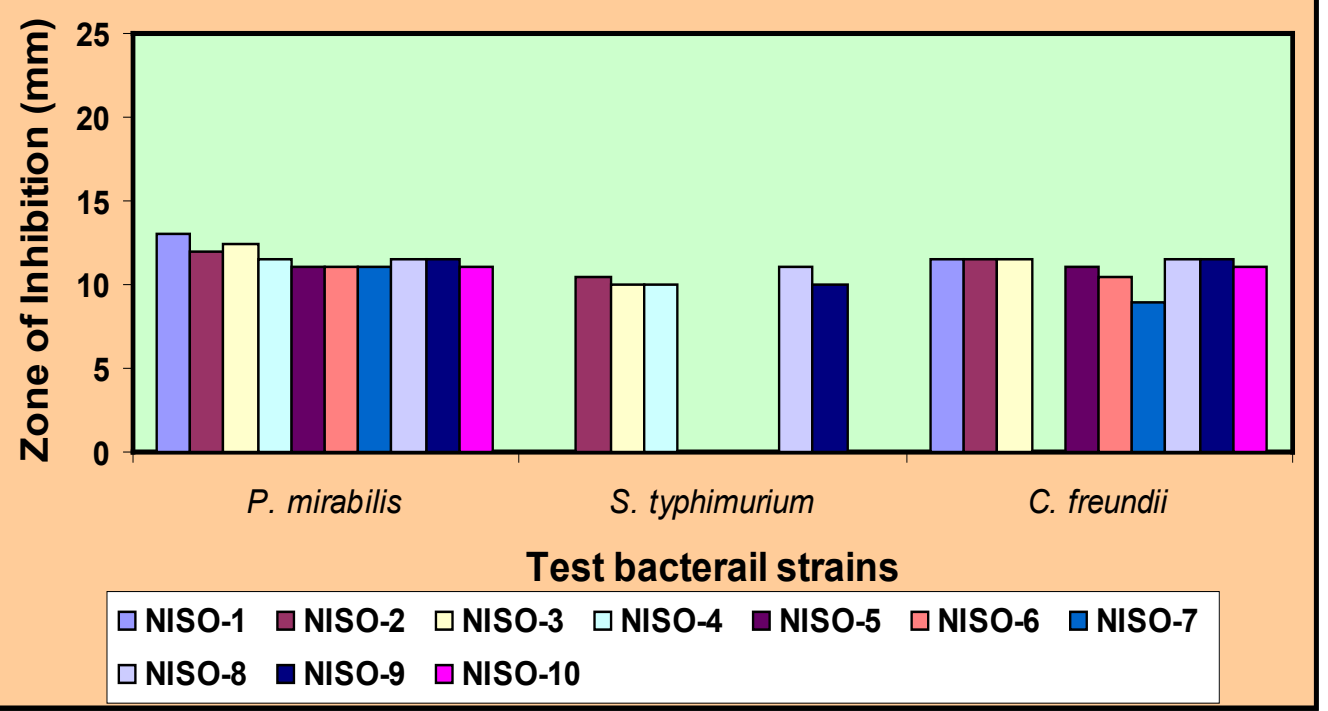

Figure 10. Antibacterial activity of isoxazoles against Gram negative bacteria in DMF.

In DMF, all compounds showed almost similar inhibition against $P$. mirabilis. Against S. typhimurium, NISO-8 show maximum activity followed by NISO-2 whereas NISO-3, NISO-4 and NISO-9 show similar minimum activity while NISO-1, NISO-5, NISO-6, NISO-7 and NISO-10 shows no activity. Against $C$. freundii, all the compounds show almost similar average activity except NISO-4. Thus, all substitutions are effective for $C$. freundii except NISO-4.

\section{Discussion}

\section{Cyanopyridines:}

The results indicate that inhibition depends on three S: solvent, strain and structure. All the compounds have the same central moiety but different substitutions as given in Table 1 . Comparison of inhibition against Gram positive bacteria of studied compounds in DMF and DMSO solvents shows that DMF is good solvent.

In DMSO, against B. cereus except NCP-4, other compounds exhibited inhibition. Table 1 shows that NCP-4 contains nitro group at para position. Thus, presence of nitro group could not affect this bacterial strain. The compound NCP-8 containing chloro group at para position is most effective. Against $M$. flavus and $S$. aureus, not a single compound is effective.

However, in DMF, again NCP-8 containing chloro group at para position exhibited maximum inhibition against $B$. cereus and $S$. aureus. Against $M$. flavus, hydroxyl group at ortho position (as in NCP-9) and only hydrogen (as in NCP-10) exhibited inhibition to the same compound. Other substitutions had no effect at all.

Thus, overall p-chloro substitution (as in NCP-8) is most effective against studied Gram positive bacteria.

In case of Gram negative bacteria, in DMSO, against $S$. typhimurium and $C$. freundii, studied compounds had no effect. However, for P. mirabilis only NCP-3, NCP-4 and NCP-8 containing bromo, nitro and chloro substitutions at para position respectively showed inhibition to the same extent.

In DMF, all the substitutions are found to be effective against all the three gram negative bacteria. The compound having no substitution i.e., NCP-10 is most effective against S. typhimurium whereas nitro group at meta position (as in NCP-5), hydroxyl group at para position (as in NCP6) and methyl group at para position ( as in NCP-7) are least effective. For P. mirabilis, all substitutions are almost equally effective. However, when there is no substitution group present 
(as in NCP-10), it is most effective for this bacteria. Against $C$. freundii, nitro group at meta position (as in NCP-5) is most effective whereas maximum inhibition is for NCP-5.

Overall, DMF is good solvent for Gram negative bacteria.

\section{Isoxazoles:}

Against Gram positive bacteria in DMSO, for B. cereus, amino group at ortho position is most effective whereas against $M$. flavus, only nitro group at meta position had effect. However, against S. aureus, all the substitution in studied compounds had no effect on studied bacterial strains. Thus, $S$. aureus is most resistant bacteria in DMSO.

In DMF, against $B$. cereus, all the substitution in studied compounds had effect and effect is maximum for compound containing hydroxyl group at para position (i.e., NISO-6). The bromo and nitro groups at para position had little effect.

Against M. flavus, nitro group at meta position (as in NISO-5) causes maximum inhibition which is followed by similar inhibition of methoxy (in NISO-1), bromo ( in NISO-3) and nitro group (in NISO-4) at para position. The inhibition due to other groups is comparatively less. However, amino group at para position ( as in NISO-2) and hydroxyl group at ortho position (as in NISO-9) had no effect at all. Thus, in this case m-nitro group is most effective. For S. aureus, again all compounds showed inhibition and hydroxy group at para position is most effective. The chloro group at para position and hydroxy group at ortho position had minimum effect.

Thus in DMF, overall compounds containing nitro group at meta position and hydroxy group at para position are most effective.

Against Gram negative bacteria, in DMSO only methoxy, amino and methyl groups at para positions are effective against $P$. mirabilis. Others substitutions showed no inhibition at all. For $S$. typhimurium, methoxy, nitro, hydroxyl and chloro groups at para positions had effect. Other compounds had no effect on this bacterium. Maximum inhibition is for NISO-1 containing methoxy group and minimum for NISO-8 having chloro group. Thus, for $S$. typhimurium, p-methoxy substitution is most effective. No inhibition is observed against $C$. freundii by any compounds. Thus, in DMSO, C. freundii is most resistant bacteria.

In DMF, all compounds showed almost similar inhibition against $P$. mirabilis. Against $S$. typhimurium, chloro group at para position (as in NISO-8) causes maximum inhibition which is followed by amino group at para position (as in NISO-2). The bromo (as in NISO-3), nitro (as in NISO-4) and hydroxyl at ortho position (as in NISO-9) had similar minimum inhibition while other groups had no effect. Against $C$. freundii, all substitutions are effective for $C$. freundii except pnitro (as in NISO-4). Thus, in DMF, $S$. typhimurium is most resistant bacteria.

\section{Conclusions}

Cyanopyridine and isoxazole derivatives were synthesized by using Vilsmeier-Haack reagent. The structures of synthesized compounds were supported by spectroscopic techniques. The antibacterial screening of these compounds shows that inhibition depends on solvent, bacterial strain and compound structure. For both Cyanopyridine and isoxazole derivatives, DMF is good solvent. Against different bacterial strains, different compounds have different effect. However, overall, S. aureus, M. flavus and C. freundii are the most resistant bacteria in the studied solvents for these synthesized compounds.

\section{Conflict of Interest}

Authors have no conflict of interest.

\section{Acknowledgement}

Authors are thankful to Heads of Chemistry and Bioscience Departments for providing necessary facilities. 


\section{References}

[1] G. Bringmann, Y. Reichert, V. Kane, The total synthesis of streptonigrin and related antitumor antibiotic natural products, Tetrahedron. 60 (2004) 3539-3574.

[2] J.P. Michael, Quinoline, quinazoline and acridone alkaloids, Nat. Prod. Rep. 14 (1997) 605618.

[3] P. Barraja, P. Diana, A. Montalbano, Pyrrolo [3,4-h] quinolinones a new class of photochemothera peutic agents, Bioorg. Med. Chem. 19(7) (2011) 2326-2341.

[4] M.P. Maguire et al., A new series of PDGF receptor tyrosine kinase inhibitors: 3-substituted quinoline derivatives, J. Med. Chem. 37(14) (1994) 2129-2137.

[5] B. Kalluraya, S. Sreenivasa, Synthesis and pharmacological properties of some quinoline derivatives, IL Farmaco. 53(6) (1998) 399-404.

[6] G. Roma et al., 1,8-Naphthyridines IV. 9-Substituted N,N-dialkyl-5-(alkylamino or cycloalkylamino) $[1,2,4]$ triazolo[4,3-a] $[1,8]$ naphthyridine-6-carboxamides, new compounds with anti-aggressive and potent anti-inflammatory activities, Eur. J. Med. Chem. 35(11) (2000) 1021-1035.

[7] Y.L. Chen et al., Synthesis and antibacterial evaluation of certain quinolone derivatives, J. Med. Chem. 44(14) (2001) 2374-2377.

[8] M.M. Ghorabi, M.S. Bashandy, M.S Alsaid, Novel thiophene derivatives with sulfonamide, isoxazole, benzothiazole, quinoline and anthracene moieties as potential anticancer agents, Acta Pharm. 64(4) (2014) 419-431.

[9] X. Zhang, A.S. Shetty, S.A. Jenekhe, Electroluminescence and photophysical properties of polyquinolines, Macromolecules. 32(22) (1999) 7422-7429.

[10] S.A. Jenekhe, L. Lu, M.M. Alam, New conjugated polymers with donor-acceptor architectures: synthesis and photophysics of carbazole-quinoline and phenothiazine -quinoline copolymers and oligomers exhibiting large intramolecular charge transfer, Macromolecules. 34(21) (2001) 7315-7324.

[11] A. Marella et al., Quinoline: A versatile heterocyclic, Saudi Pharma. J. 21(1) (2013) 1-12.

[12] S. Kumar, S. Bawa, H. Gupta, Biological activities of quinoline derivatives, Mini Rev. Med. Chem. 9(14) (2009) 1648-1654.

[13] K.R.A. Abdellatif et al., Design, synthesis and biological evaluation of new 4-(4-substitutedanilino) quinoline derivatives as anticancer agents, Med. Chem. Res. 26(5) (2017) 929-939.

[14] B.C. Revanasiddappa et al., Synthesis and biological studies of some novel schiff bases and hydrazones derived from 8-hydroxy quinoline moiety, Int. J. Chem. Tech. Res. 1 (2009) 1100-1104.

[15] Z.G. Luo et al., Synthesis and biological activities of quinoline derivatives as HIV-1 integrase inhibitors, Chem. Res. Chin. Univ. 25 (2009) 841-845.

[16] S. Vandekerckhove, M. D'hooghe, Quinoline-based antimalarial hybrid compounds, Bioorg. Med. Chem. 23(16) (2015) 5098-5119.

[17] X.F. Zhu et al., Synthesis and structure-activity relationships study of $\alpha$-aminophosphonate derivatives containing a quinoline moiety, Chin. Chem. Lett. 28(7) (2017) 1514-1518.

[18] S. Kirankumar et al., New efficient synthesis of 3-carboxylquinolines, J. Korean Chem. Soc. 56(3) (2012) 322-327.

[19] X. Franck et al., Biological evaluation of substituted quinolones, Bioorg. Med. Chem. Lett. 14(14) (2004) 3635-3638. 
[20] H. Amii, Y. Kishikawa, K. Uneyama, Rh(I)-catalyzed coupling cyclization of n-aryl trifluoroacetimidoyl chlorides with alkynes: one-pot synthesis of fluorinated quinolones, Org. Lett. 3(8) (2001) 1109-1112.

[21] T. Igarashi et al., One-pot synthesis of substituted quinolines by Iridium-catalyzed threecomponent coupling reaction, Chem. Lett. 34(1) (2005) 106-107.

[22] N. Sakai et al., Yb(OTf)3-catalyzed cyclization of an N-silylenamine with 2-methylene-1,3cyclohexanedione to afford a 7,8-dihydroquinolin-5(6H)-one derivative and its application to the one-pot conversion to a 2,3,5-trisubstituted quinoline derivative, Tetrahedron Lett. 47(8) (2006) 1261-1265.

[23] K.D. Thomas, A.V. Adhikari, N.S. Shetty, Design, synthesis and antimicrobial activities of some new quinoline derivatives carrying 1, 2, 3-triazole moiety, Eur. J. Med. Chem. 45(9) (2010) 3803-3810.

[24] V.V. Kouznetsov, L.Y. Me'ndez, C.M.M. Gómez, Recent progress in the synthesis of quinolines, Curr. Org. Chem. 9(2) (2005) 141-161.

[25] A. Lilienkampf et al., Structure-activity relationships for a series of quinoline-based compounds active against replicating and non replicating Mycobacterium tuberculosis, J. Med. Chem. 52(7) (2009) 2109-2118.

[26] Q. Guo et al., Synthesis of quinoline derivatives from anilines and aldehydes catalyzed by $\mathrm{Cp}_{2} \mathrm{ZrCl}_{2}$ and recyclable $\mathrm{Cp}_{2} \mathrm{ZrCl}_{2} / \mathrm{MCM}-41$ system, Catalysis Today. 263 (2016) 117-122.

[27] R.D. Chambers et al., Elemental fluorine: Part 15. Selective direct fluorination of quinoline derivatives, J. Fluorine Chem. 125(5) (2004) 661-671.

[28] R. Jain et al., Synthesis of amino acid derivatives of 8-[(4-amino-1-methylbutyl)amino]-6methoxy-4-substituted/4,5-di substituted-quinolines as potential antimalarial agents, Ind. J. Chem. 33(3) (1994) 251-254.

[29] M. Croisy-Delcey et al., Diphenyl quinolines and isoquinolines: synthesis and primary biological evaluation, Bioorg. Med. Chem. 8(11) (2000) 2629-2641.

[30] B.N. Acharya, D. Thavaselvam, M.B. Kaushik, Synthesis and antimalarial evaluation of novel pyridine quinoline hybrids, Med. Chem. Res. 17(8) (2008) 487-494.

[31] A.H. Abadi, R. Brun, Synthesis and evaluation of novel 7-trifluoromethyl-4-(4-substituted anilino)quinolines as antiparasitic and antineoplastic agents, Arzneimittelforschung Drug Research. 53(09) (2003) 655-663.

[32] R. Subashini, S. Mohana Roopan, F.N. Khan, Synthesis and free radical scavenging property of some quinoline derivatives, J. Chil. Chem. Soc. 55(3) (2010) 315-317.

[33] L. Hu et al., Design, Practical synthesis, and biological evaluation of novel 6(pyrazolylmethyl)-4-quinoline-3-carboxylic acid derivatives as HIV-1 integrase inhibitors, Molecules. 17(9) (2012) 10652-10666.

[34] N. Sun et al., Antibacterial activity of N-methylbenzofuro[3,2-b]quinoline and Nmethylbenzoindolo[3,2-b]-quinoline derivatives and study of their mode of action, Eur. J. Med. Chem. 135 (2017) 1-11.

[35] J. A. Riddick, W. B. Bunger, T. Sakano, Organic solvents: physical properties and methods of purification, Techniques of Chemistry, New York, 1986.

[36] C. Perez, M. Pau, P. Bazerque, An antibiotic assay by agar well diffusion method, Acta Biol. Med. Exp. 1591) (1990) 113-115.

[37] S. Baluja, S. Chanda, Pyrazoline derivatives: Synthesis and antibacterial studies, World. Res. J. Biochem. 1(1) (2012) 6-10. 